\title{
ATMOSPHERIC INFRASONIC MONITORING AND ANALYZING OF THE INFRASONIC EVENTS BEFORE THE 2015 NEPAL EARTHQUAKE
}

\author{
Riheng Huang ${ }^{a}$, Li Zhang, Jun Yang, Bingyi Zhang, Jing Zhao, Jia Xu \\ Changcheng Institute of Metrology \& Measurement, Beijing 100095, China \\ anihong.real@foxmail.com
}

\begin{abstract}
Keywords: atmospheric infrasound, monitoring, location, Nepal earthquake
Abstract. One infrasonic monitoring network in east-central China is built to study the infrasonic phenomenon caused by spontaneous or artificial events in the atmosphere. Two events of anomalous infrasound waves were recorded at five infrasonic monitoring stations before the Nepal earthquake which happened on 24 April 2015. Sources of the infrasonic events are located by using the methods of minimum variance based on the infrasonic time delays. A novel method is shown for calculating the velocity projected surface and the evidence of spatial correlation between the source and earthquake is produced in this paper.
\end{abstract}

\section{Introduction}

It has been suggested that infrasound can be caused by numerous events, for instance typhoon, volcanic eruption, meteor, aurora, earthquake [1]. Some researches indicate that long duration infrasound whose frequency is between $0.001 \mathrm{~Hz}$ and $0.01 \mathrm{~Hz}$ can be monitored 10 days before some of the earthquakes. This kind of infrasound is known as the precursory infrasound.

The previous study of the mentioned phenomena mainly focused on the correlation of time. In 1975, Gossard, E. E., Hooke,W.H. from America studied on the characteristics of the significant waves in the air, then proposed the hypothesis of using infrasound to predict earthquake [2]. Li Junzhi statistically analyzed the earthquakes happened in 1995 with $\mathrm{M}_{s} \geq 6$. The result shows that nearly $78 \%$ of earthquakes lead to precursory infrasound. Cui Xiaoyan did the same research with earthquakes $\left(M_{s} \geq 7\right)$ which occurred between 2002 to 2009, and 57\% earthquakes have precursory infrasound.

It is significant to seek the acoustic source of the infrasound on account of there is less evidence of the beforehand precursory caused by earthquakes. The only one instance found is that Yang Yichun positioned the Lushan earthquake and Pakistan earthquake happened in 2013. There was some lag between the positioning result and the epicenter [3].

In this paper, two events of the infrasonic signals are shown, one of which monitored separately at around 5 April 2015.The other was found at 18 April 2015 eight days before Nepal earthquake $\left(M_{s}=7.1\right)$. Then the two speeds of infrasound traveling projected to earth surface in both cases are calculated. Finally, the sources of the two events are position by the method of minimum variance.

\section{Infrasound Monitoring Network}

Up until now, twelve stations of monitoring infrasound have been established which are set up in Beijing, Tianjin, Zhangjiakou, Nanjing, Wuxi, Taizhou, Chongqing, Chengdu, Mianyang, Yibin, Xiamen. Conditions of distribution of station are shown in Fig. 1. It is found that the distribution has a great influence on the correlation of monitoring data [4-5]. 


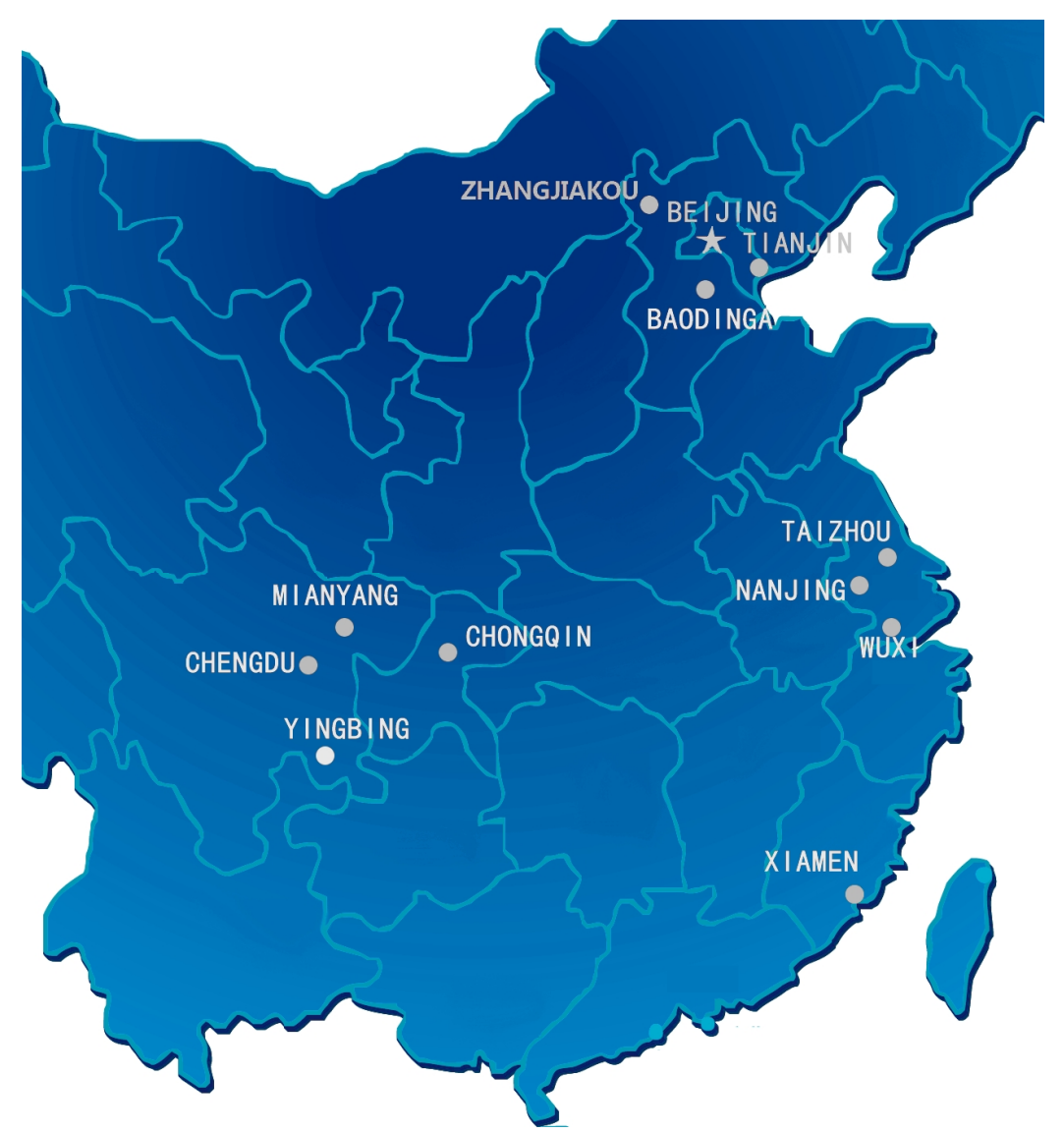

Fig. 1 Distribution of monitoring infrasound station

\section{Positioning Methods}

The infrasonic source can be located by a mathematical method ideally based on the received signals.

Firstly, the degree of correlation between the signals should be assessed by correlation coefficient. If the coefficient is high enough, it is necessary to calculate time delay by correlation of the two signals.

Then location of the source of infrasound can be estimated by the minimum variance of time delay. Traversing each point around map, the variance is calculated by the Eq. 1 .

$$
s=\sum\left(\frac{D_{i}-D_{j}}{v}-t_{i, j}\right)^{2}
$$

Where $s$ is the variance of the point; $D_{i}, D_{j}$ represent the distance from the point chosen to the station $i$ or station $j ; v$ is the speed of infrasound traveling projected to earth surface; $\mathrm{t}_{i, j}$ means the time delay of the signals monitored by station $i$ and station $j . v$ is unknown here. According to previous research the speed is about $10 \mathrm{~m} / \mathrm{s}^{2}$. Therefore, it is essential seeking the optimal speed to make the variance minimum.

Finally, location of the infrasonic source can be found around from the distribution of variance. Smaller the variance of the point more possible the source is the point.

\section{Observation and Analysis}

It is uncommon from the observation of the past year that the infrasound of frequency is between $0.001 \mathrm{~Hz}$ and $0.01 \mathrm{~Hz}$ whose duration is around half hours to 4 hours with large amplitude. But during the ten days the precursory infrasound which has been monitored always exist one or more earthquake. The similar signals of infrasound can be monitored by several stations, and closer the two stations are more similar the signals are. 


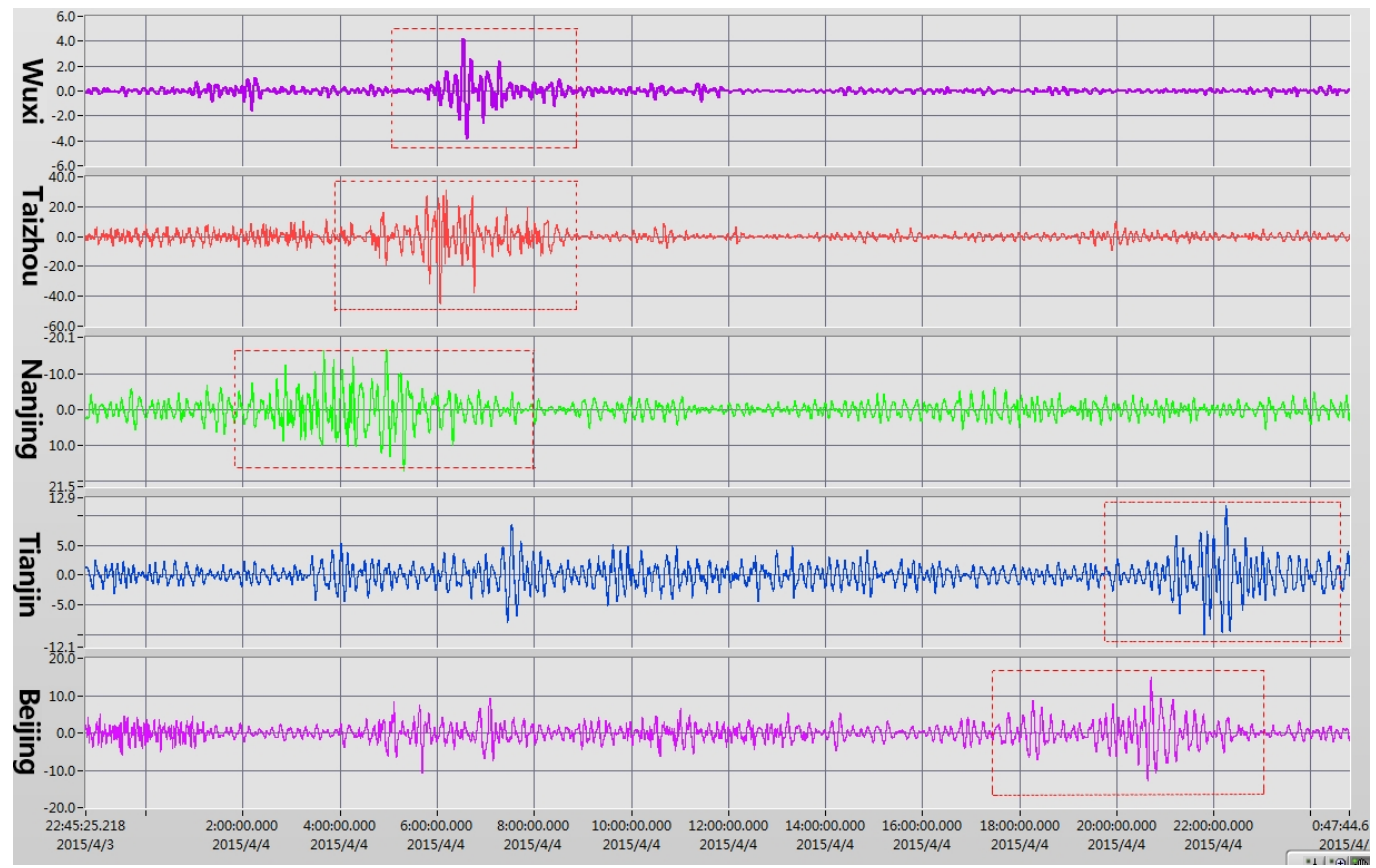

Fig. 2 Infrasonic signals at around 4 April 2015

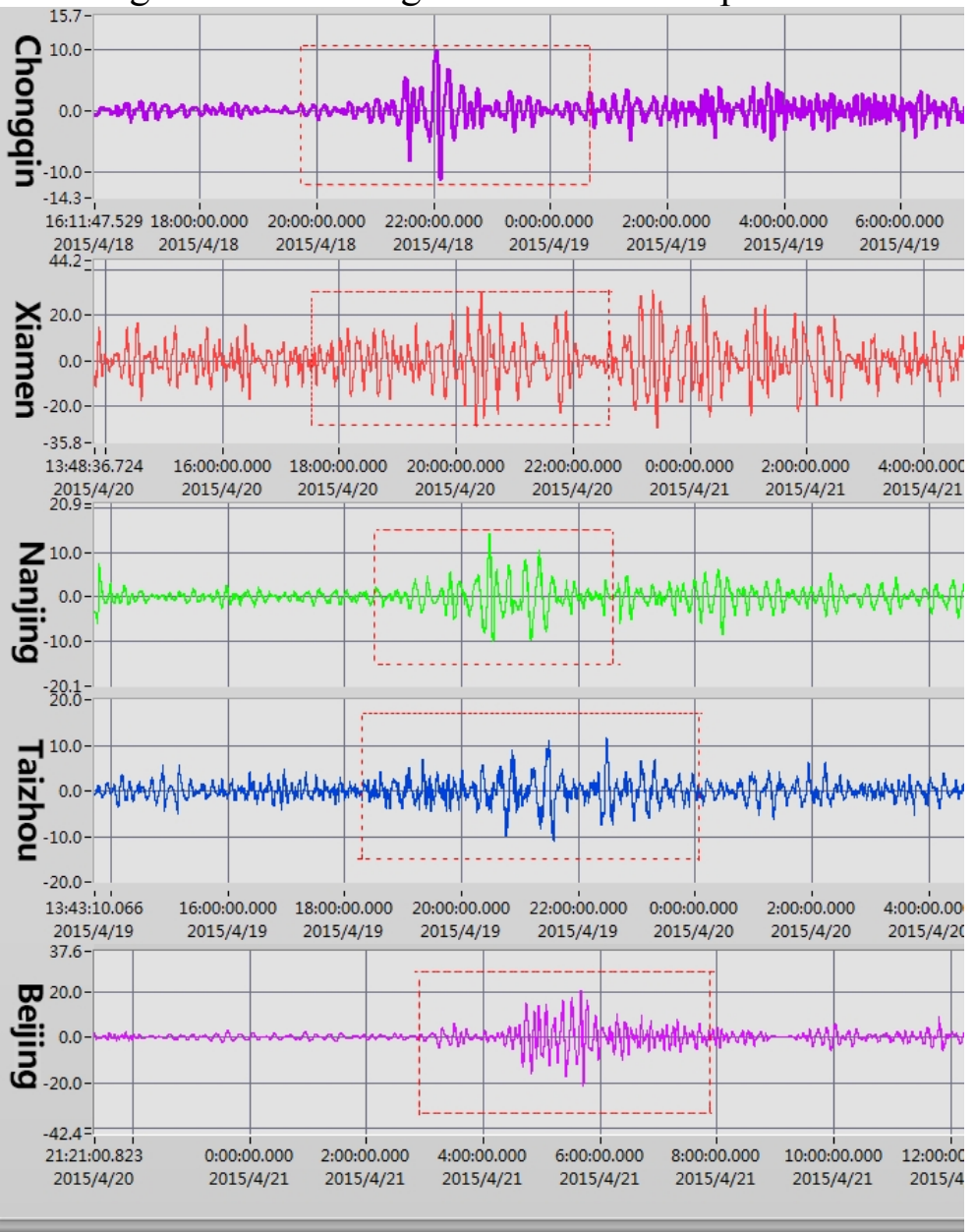

Fig. 3 Infrasonic signals at around 17 April 2015

The Fig. 4 and Fig.5 are figures of the relationship between the speed and minimum variance respectively calculated based on time delay of signals in the Fig.2 and Fig.3 using the methods mentioned. The minimum variance here is calculated by traversing over all points on the map under the specific velocity of the infrasound. In the Fig.4, minimum variance of time delay is the smallest one when $v=6.3 \mathrm{~m} / \mathrm{s}$. For the Fig. 5 the minimum speed is $v=6.1 \mathrm{~m} / \mathrm{s}$. 


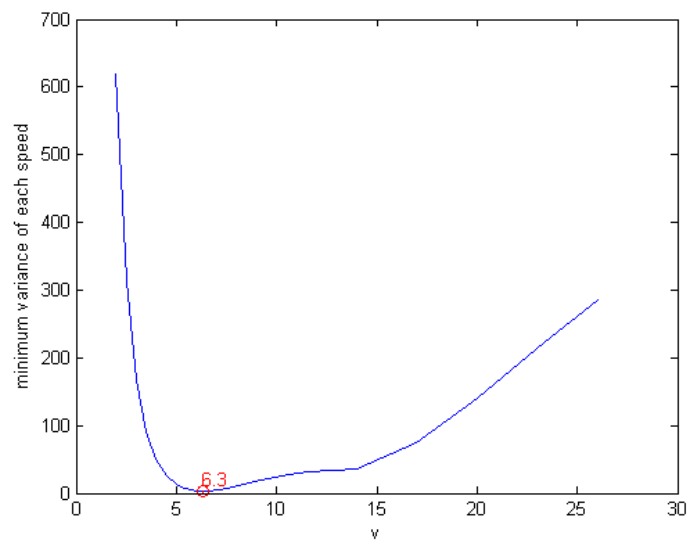

Fig.4 relationship between speed and minimum variance based on the signals in the Fig.2

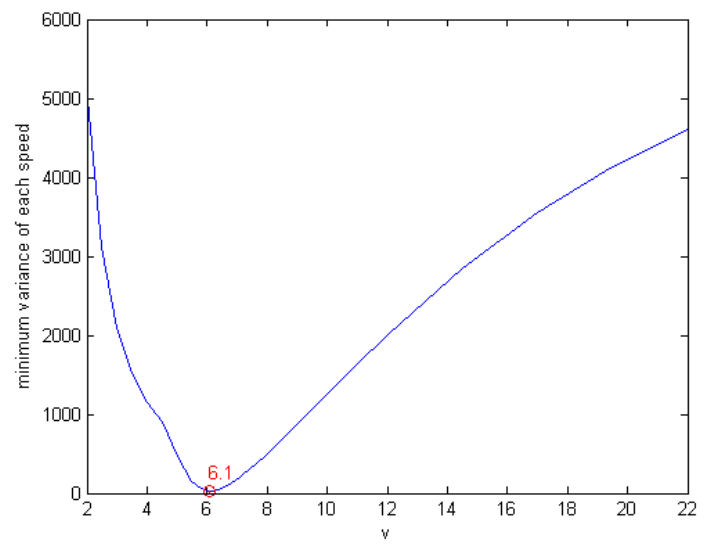

Fig.5 Relationship between speed and minimum variance based on the signals in the Fig. 3

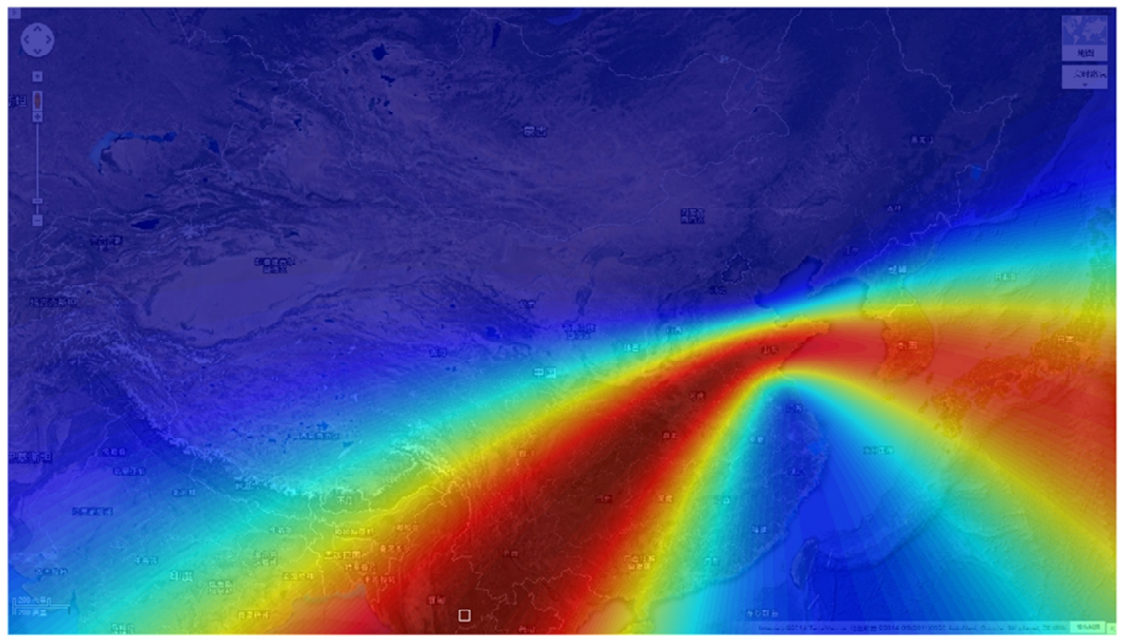

Fig.6 Estimation of the source of the signals in the Fig. $2(v=6.3 \mathrm{~m} / \mathrm{s})$

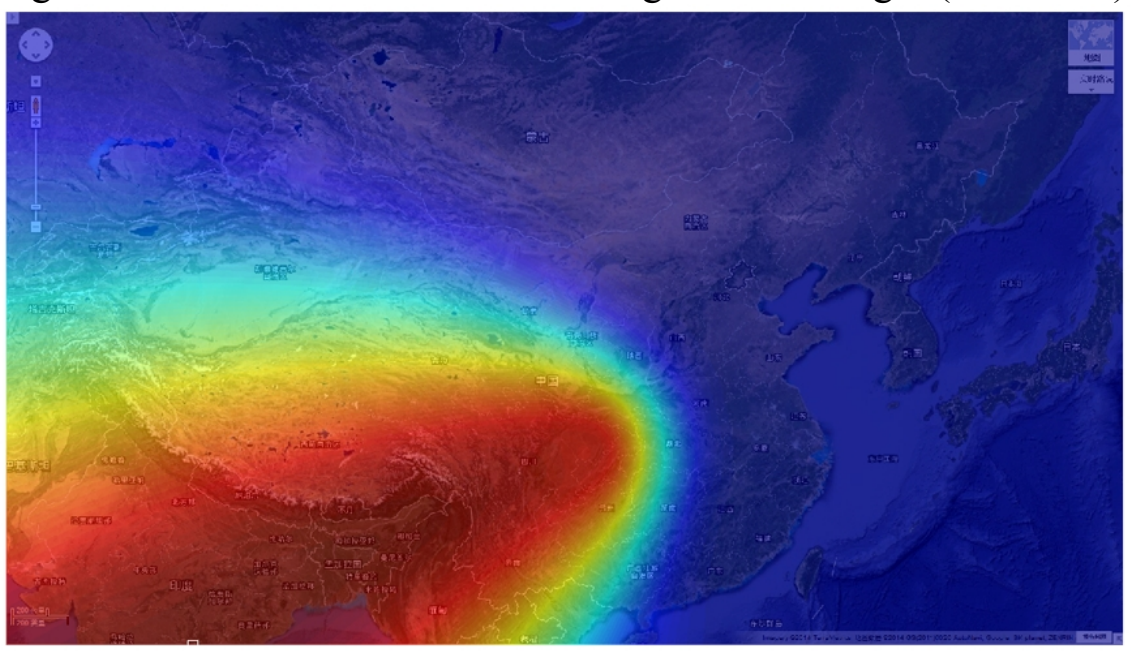

Fig.7 Estimation of the source of the signals in the Fig. $3(v=6.1 \mathrm{~m} / \mathrm{s})$

Fig. 6 and Fig.7 are the distribution maps of variance of time delay when speed of infrasound traveling projected to earth surface is the speed of minimum variance. Variances of each point in map are shown in the Fig. 6 or Fig. 7, red stands for a smaller variance, while blue stands for the opposite. White squares are the points whose variance is the smallest one.

On the basis of geometric method and the signals of Wuxi, Taizhou, Nanjing at around 4 April 2015, the azimuth angle of infrasonic source is South 63.6 degrees west in Nanjing. The direction is similar to the point of minimum variance. There was an earthquake occurred in Yunnan Province at April 13 which is nine days after the signals of Fig. 2 recorded. The epicenter of this earthquake is approximate 
to the white square in Fig.6. This means that the source of infrasound was more likely engendered by the earthquake. But during the ten days there also was one earthquake which is violent enough in Inner Mongolia. Its infrasound might be blended with infrasound from the source mentioned before. It is further research to separation the signals observed.

During the time from April 18 to April 28, Nepal earthquake which might be the most severe earthquake in this year was in the red district of Fig. 7. The epicenter is in the red area in the Fig.7. The deviation between the white square and epicenter may be the result of assuming constant propagation of infrasound.

\section{Conclusions}

The infrasound discussed in this paper whose frequency around $0.001 \mathrm{~Hz}$ to $0.01 \mathrm{~Hz}$ is often observed ten days prior to earthquake happen. The two events selected are considerably ordinary infrasonic anomalies in the past year. It is innovative that using minimum variance of time delay to estimate the speed of infrasound traveling projected to earth surface. Then the probability area of the infrasonic source is calculated. More tests are required to the method because of approximately half stations were built recently. The method of minimum variance only used the signals from less than six stations.

Choosing the similar infrasonic signals is a difficult thing, although it is mentioned in this paper. Judging whether the infrasound monitored is precursory infrasound, and determining how to correspond two signals from different stations. To complicate matters further, there may be may several infrasonic sources around at the same time. It is inevitable that infrasound from diverse sources is monitored and blended into signal in any station.

Further research can be surrounding extracting the infrasonic sources from signals for the sake of improving accuracy of time delay to reduce the influence of several sources. Then pay more attention to the principle of infrasonic spreading. Dissemination of infrasonic can be influenced by so many element that there is no mathematical model to describe it.

\section{References}

[1] Evers, L.G., and H. W. Haak. The Characteristics of Infrasound, its Propagation and Some Early History. Infrasound Monitoring for Atmospheric Studies. Springer Netherlands, 2009:3-27.

[2] Gossard, E. E., et al. "Waves in the atmosphere." Geophysical Research letters 9.5(1982):575-578.

[3] Lü, Jun, et al. "Anomalous Infrasonic Waves Before a Small Earthquake in Beijing." Chinese Journal of Geophysics 55.5(2012):580-586.(In China)

[4] Christie, D. R., and P. Campus. The IMS Infrasound Network: Design and Establishment of Infrasound Stations. Infrasound Monitoring for Atmospheric Studies. Springer Netherlands, 2009:29-75.

[5] Brachet, Nicolas, et al. "Monitoring the Earth's Atmosphere with the Global IMS Infrasound Network." Infrasound Monitoring for Atmospheric Studies (2009):10773. 